\title{
Miscibility in Two Blend Systems of Homologous Semicrystalline Aryl Polyesters Involving Poly(trimethylene terephthalate)
}

\author{
Yu-Hsiao KUO and Ea Mor WoO ${ }^{\dagger}$ \\ Department of Chemical Engineering, National Cheng Kung University, Tainan 701-01, TAIWAN
}

(Received August 19, 2002; Accepted November 27, 2002)

\begin{abstract}
The miscibility in the pairs of homologous series of semicrystalline polyesters: poly(trimethylene terephthalate) (PTT) and poly(ethylene terephthalate) (PET) and poly(trimethylene terephthalate) (PTT) and poly(butylene terephthalate) (PBT), respectively, were investigated as model systems. In this study, two binary blend systems each containing two semicrystalline aryl-polyesters differing by the number of methylene units, were found to be fully miscible (in absence of trans-esterifications) in accordance with criteria of microscopy morphology and thermal transition. In addition, only a single composition-dependent $T_{\mathrm{cc}}$ was taken as a valid supportive evidence for miscibility in blends of two crystallizable polymers. The claimed miscibility applied to the quenched state of the blends, and not the crystallized domain involving the crystal cells in the crystalline regions. The crystal morphology evidence showed that the unit cell types in the miscible blends remained individually different, and co-existed in common spherulites.

KEY WORDS Poly(trimethylene terephthalate) (PTT) / Poly(ethylene terephthalate) (PET)/

Poly(butylene terephthalate) (PBT) / Miscibility /
\end{abstract}

Miscibility is not always common in the blends of homologous polymers that differ in structures but possess the same functional groups. Studies of polymer miscibility have been more commonly focused on polymers of different structures, especially those possessing potential sites for specific interactions. By comparison, less has been reported on the phase behavior of blends comprising polymers with similar or homologous structures. Conventional notions have been that a slight offset in the chemical structures of the constituent polymers may lead from miscibility to phase separation, or vise versa, in the mixtures that comprise the polymers pairs whose structures are similar but offset by a simple chemical unit. Consequently, miscibility in polymers with similar structures has been an interesting subject of studies. It has been known that polymers of similar chemical structures (e.g., homologous series of polymers) are not always likely to form a miscible mixture. In general, polymers in homologue series differing by a methylene or methyl unit are usually not miscible, which is especially true for polymers that do not exhibit any polar forces or specific interactions. For examples, high-density polyethylene (HDPE) is generally not miscible with low-density polyethylene (LDPE), which differs from the former only by random and occasional pendant $\mathrm{CH}_{3}$ groups in the main chains. It is also classically known that polyethylene $\left(-\left[\mathrm{CH}_{2}-\mathrm{CH}_{2}\right]-\right)$ and polypropylene (- $\left.\left[\mathrm{CH}_{2}-\mathrm{CH}\left(\mathrm{CH}_{3}\right)\right]-\right)$, which differ from each other only by a pendant methyl group in the repeat unit, are not miscible. Further examples are abundant in recent papers in the literature. Blend systems comprising of polystyrene (PS) and poly ( $\alpha$-methyl styrene) (P $\alpha$ MS), whose repeat units differ only by a methyl group $\left(-\mathrm{H} v s\right.$. $\left.-\mathrm{CH}_{3}\right)$ in the $\alpha$-position, have been extensively studied. ${ }^{1-6}$ PS and P $\alpha$ MS of high molecular weights are generally immiscible; only PS and P $\alpha$ MS of low enough molecular weights can form blends that exhibit an upper critical solution temperature (UCST) behavior. This means that PS and P $\alpha$ MS of low-molecular-weights are immiscible at ambient temperature but can turn into a homogeneous phase only at elevated temperatures that depend on $M_{\mathrm{w}}$ of the polymers. On the other hand, blends of PS with P4MS, with a methyl group on the 4-positon of the phenyl ring, are known to be immiscible. ${ }^{7}$ Apparently, a slight alteration in the chemical structures, for examples an isomeric change, of polymers can significantly influence the phase behavior in the mixture forms. In yet one more example, polyoxymethylene and polyoxyethylene are not miscible. Blends of isomers, such as $\mathrm{P} 4 \mathrm{MS} / \mathrm{P} \alpha \mathrm{MS}$, have been known to be immiscible at ambient temperature, but turn to a miscible mixture and exhibit a UCST behavior only at high temperatures.

The number of miscible blends composed of both crystallizable polymers is quite small in comparison to amorphous/crystalline or amorphous/amorphous blend systems that are known in the literature. An interesting and notable example in the literature for miscible blend systems involving two crystalline polymers is given by the known case of poly(ethylene

${ }_{\dagger}^{\dagger}$ To whom correspondence should be addressed (Phone: +886-6-275-7575 Ext. 62670, Fax: +886-6-234-4496, E-mail: emwoo@mail.ncku. edu.tw). 
oxide) and poly(L-lactide) (PLLA), ${ }^{9,10}$ which is a polyether/polyester system. Another interesting example is given by polyester/polyester blend systems. Poly(ethylene terephthalate) (PET), poly(trimethylene terephthalate) (PTT), and poly(butylene terephthalate) (PBT) all belong to a series of homologous arylpolyesters. Of these, PET and PBT are more widely known as commercially important polyesters, each with unique properties as engineering resins or textile fibers, while PTT is a relatively new comer that possesses interesting balance of desired properties. Blends of PET and PBT have been more widely studied in early time, ${ }^{11-16}$ however, blends of all semicrystalline constituents involving PTT have yet to be explored. Thus, miscibility (in amorphous regions) in blends of these aryl polyesters may further help in exploiting the useful properties by combining two or three of these different aryl polyesters. The objectives of this work were to investigate the fundamental aspects of phase, morphology, crystals, and thermal behavior of binary blend systems comprising two of the homologous series of aryl polyesters, which are all semicrystalline engineering plastics and differ in the backbone units only by one or two methylene group(s). Truly miscible blends composed of any two of these three polymers differing in properties might offer a unique opportunity to develop a new polymer material with flexible combination from three constituents. Understanding of the blend's phase behavior, thermal properties and crystalline morphology can help extending the service ranges and/or processing advantages of these useful engineering polymer systems with more flexible balances of properties.

\section{EXPERIMENTAL}

\section{Materials}

Poly(ethylene terephthalate) (PET) used in this study was supplied in an additive-free form (Shin-Kong Corp., Taiwan). Poly(butylene terephthalate) (PBT) is a semicrystalline polyester, which was obtained as courtesy research material from GE Corp. (PBT-315). Poly(trimethylene terephthalate) (PTT), or alternatively named poly(propylene terephthalate) (PPT), was supplied as a courtesy sample material of a research-grade resin with no additives and was synthesized by Industrial Technology Research Institute (ITRI, Taiwan). The chemical structures of the repeating units of PET, PBT, and PTT are shown as following:

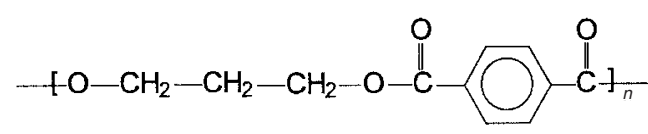

PTT

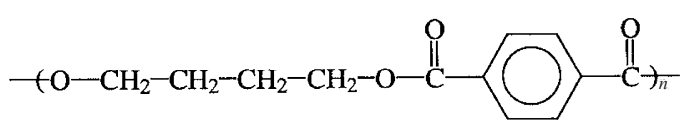

PBT

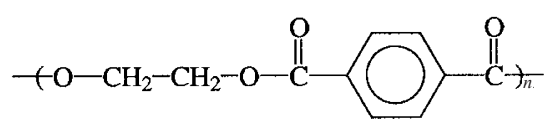

PET

Blends of polyesters were in two different methods for comparison. First ternary blend samples were prepared by solution mixing and casting from dichloroacetic acid at $60^{\circ} \mathrm{C}$. The solution mixing and preparation of blend samples ensured absence of possible transesterification reactions that might occur in polyesters at high temperatures. Second blend samples of the aryl polyesters were prepared by melt-blending at above the melting temperatures. The neat polymers were first pulverized (ground) into fine powder. This manipulation was made in order to ensure that thorough mixing could be completed within the shortest time possible, with least thermal degradation. An aluminum mold with a small mixing chamber ( $c a$. 2-g capacity) was laboratory-made and it was designed in such way that thorough blending could be completed with manual stirring. This set-up was especially ideal for handling small quantities of polymer samples ( $1-2 \mathrm{~g}$ or less per batch). During blending, dry nitrogen was maintained by continuously purging into the mixing chamber to ensure minimum thermal degradation. Heating and temperature control was provided by a hot stage with a temperature controller set at $250-300^{\circ} \mathrm{C}$. For temperature accuracy, all thermal treatments imposed on the blend samples were performed in the precisiontemperature cells of the differential scanning calorimeter. Melt-crystallization of all samples was performed by melting at $\sim 280^{\circ} \mathrm{C}$ (in DSC cells) for $5 \mathrm{~min}$, then quenched quickly to a desired isothermal temperature for a specified period of time.

Apparatus. The glass transition temperatures and other thermal transitions were measured with a differential scanning calorimeter (PerkinElmer DSC-7) equipped with an intra-cooler and a computer for data acquisition/analysis. Unless otherwise specified, all $T_{\mathrm{g}}$ measurements were made at a scan rate of $20^{\circ} \mathrm{C} \mathrm{min}^{-1}$. All $T_{\mathrm{g}}$ values were taken as the onset temperature of the transition (the change of the specific heat) in the DSC thermograms. Prior to $T_{\mathrm{g}}$ characterization, all samples, sealed in pans, had been melted and quenched to fully amorphous states. Direct quenching in liquid nitrogen with subsequent drying of moisture was found to yield the best result.

An optical light microscope (Nikon Optiphot-2, 
POL) was used for preliminary observation of phase behavior of blends in rapid-quenched amorphous states. Optical appearance and phase changes in the blends were inspected at step-wise elevated temperatures up to where degradation occurred $\left(\sim 300^{\circ} \mathrm{C}\right)$ in order to monitor whether or not there existed a cloud-point transition. In addition, the crystalline morphology of the blends was also examined with polarized-light microscopy. The blends, pressed into thin films between two glass slides, were placed on the microscopic heating stage (Linkam THMS-600 with TP-92 temperature programmer) for temperature control. Morphology (fracture surfaces) of blends (in quenched amorphous states) was examined using a scanning electron microscope (SEM, Model JEOL JXA-840). The blend film samples for scanning electron microscopy were solution-cast to be thick enough so that fracture surface of the thickness (cross section) could be conveniently examined. The fractured samples were coated with gold by vapor deposition using a vacuum sputterer.

Wide-angle X-Ray instrument (WAXD) was Shimadzu XRD-6000 with copper $\mathrm{K}_{\alpha}$ radiation $(40 \mathrm{kV}$ and $45 \mathrm{~mA}$ ) and a wavelength of $1.542 \AA$. The scanning angle ranged $2 \theta=5^{\circ}$ to $35^{\circ}$, with a step scanning of $2^{\circ}$ for $1 \mathrm{~min}$. Thermal treatments of blend samples for $X$-Ray diffraction or SEM characterization were performed in the DSC cells for precise temperature control of intended thermal treatments.

\section{RESULTS AND DISCUSSION}

\section{PTT/PBT Blend System}

The blend samples (after being rapid-quenched to amorphous states to depress the crystallinity) were visually and optically clear, and free of any visibly heterogeneous domains, as observed using the OM technique. Possible effect of temperature on blend phase transition (e.g., from miscibility to phase separation or vise versa) was examined using the optical microscope. No cloud point transition (from clarity/homogeneity to cloudiness/phase separation) was found in any blend compositions, suggesting that these blends remained optically clear in quenched state or in melt state and no changes in the phase behavior were noticed within the wide temperature range investigated (ambient to slightly above $300^{\circ} \mathrm{C}$ ).

Thermal analysis was performed to reveal the thermal transitions of these blends. Figure 1 shows the DSC thermograms $\left(20^{\circ} \mathrm{C} \mathrm{min}^{-1}\right)$ revealing only a single $T_{\mathrm{g}}$ (arrow-marked) for each of the PTT/PBT blend samples of 11 different compositions as indicated on the DSC traces. The single $T_{\mathrm{g}}$ for each of the PTT/PBT blend samples is seen to increase steadily with com-

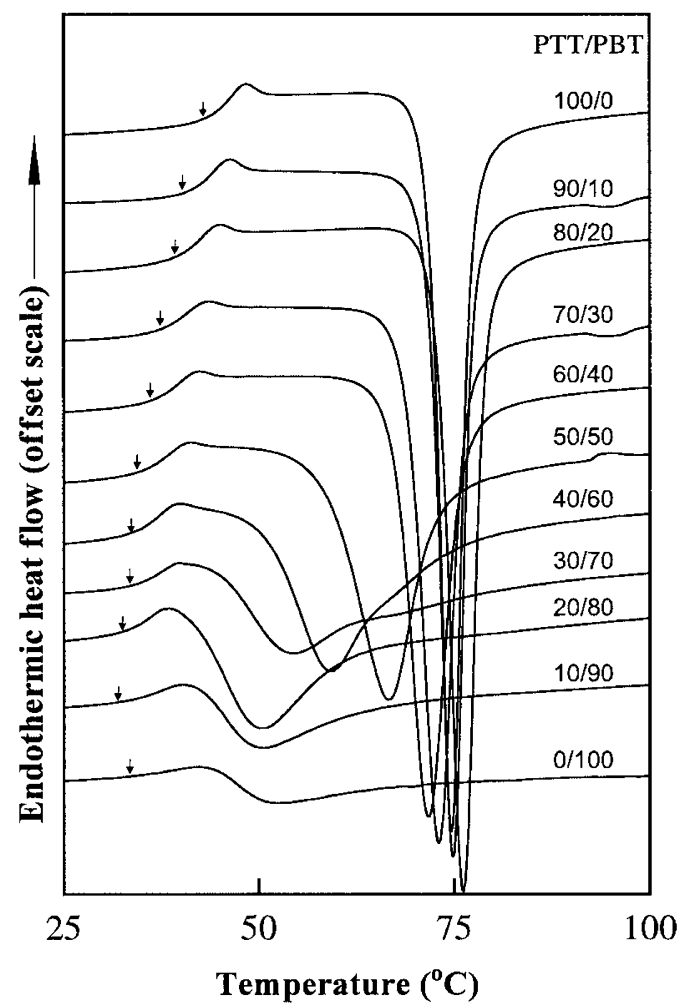

Figure 1. DSC thermograms for PTT/PBT blend samples of 11 different compositions.

position changes, i.e., it increases with the increase of the higher- $T_{\mathrm{g}}$ PTT content in the blend. Note that the $T_{\mathrm{g}}$ signal of the neat PBT is not quite apparent and its temperature location is hard to define owing to difficulty in quenching the neat PBT into completely amorphous material. In addition, a cold-crystallization exotherm is apparent for all blend compositions. The cold-crystallization exotherm (whose peak labeled as $T_{\mathrm{cc}}$ ) was detected because the blends were quenched into amorphous states prior to DSC scanning. Subsequently, upon scanning to above $T_{\mathrm{g}}$ 's, the originally amorphous chains of the semi-crystalline polymers (both PBT and PTT) quickly re-organized into ordered chains of crystal. There are two interesting trends for the crystallization exotherm of the blends, which varies systematically with respect to the compositions. The first trend is that the crystallization exotherm peak increases with the increase of PTT contents and the second trend is that the peak width gradually changes from the wider characteristic of PBT to narrow characteristic of PTT. Interestingly, these two semi-crystalline polymer chains in the blend seem to crystallize simultaneously upon heating, showing only one exotherm peak. These phenomena further indicate that the polymers chain segments in the mixtures were likely intimately mixed in fine segmental scales and these two intimately mixed chains act as there is only a single $T_{\mathrm{g}}$ in responding to external thermal changes. As a result, these 


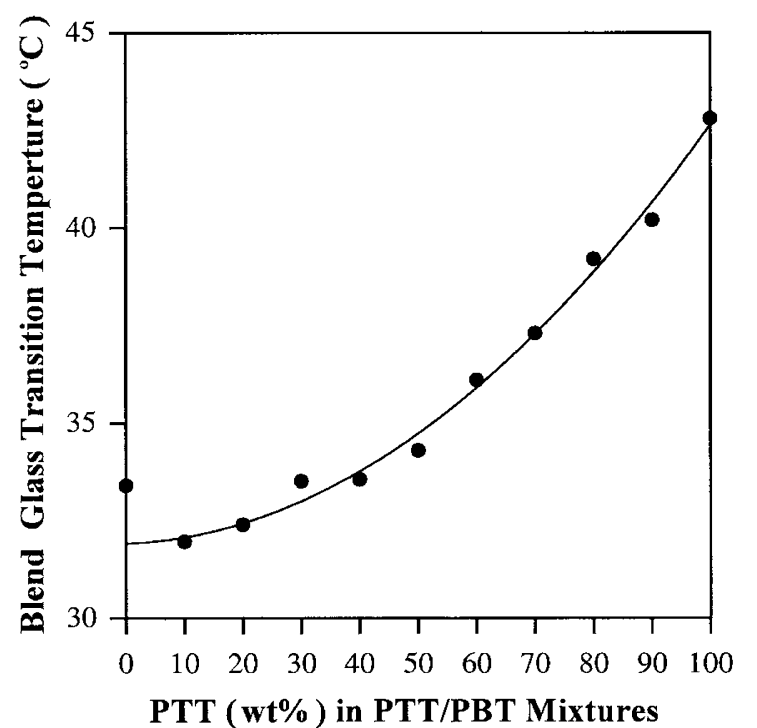

Figure 2. Plot of $T_{\mathrm{g}} v s$. composition for PTT/PBT blend system.

two different polymer chains responded to temperature changes in concerted ways and re-organized simultaneously; otherwise, there would have been two crystallization exotherm peaks. All these features suggest that the polymer chains are mixed in such fine molecular/segmental scales that the thermal responses of the mixtures act as averages of these two different polymers.

Figure 2 shows a plot of $T_{\mathrm{g}} v s$. composition relationship for the PTT/PBT blend system. To examine the quantitative trend of $T_{\mathrm{g}}$ variation with composition, the onset $T_{\mathrm{g}}$ of each blend was plotted as a function of weight fraction and fitted with the Gordon-Taylor (G$\mathrm{T})$ equation: ${ }^{17} T_{\mathrm{g}}=\left(\omega_{1} T_{\mathrm{g} 1}+\mathrm{k} \omega_{2} T_{\mathrm{g} 2}\right) /\left(\omega_{1}+\mathrm{k} \omega_{2}\right)$, where $\omega_{\mathrm{i}}$ is the mass fraction of polymer component $\mathrm{i}$, and $\mathrm{k}$ is a fitting parameter. A fitted value of $\mathrm{k}=0.35$ was obtained. The experimental $T_{\mathrm{g}}$-composition relationship for this system as shown in this figure suggests that this is a miscible blend system, with reasonable but not particularly strong/specific interactions.

Multiple, or partially overlapped, $T_{\mathrm{g}}$ 's, if any, can be better resolved by such treatments. Physical aging (annealing at below but near $T_{\mathrm{g}}$ ) was used to enhance the $T_{\mathrm{g}}$ resolution in assessing the thermal criteria of miscibility. In addition, such treatment was useful for discerning any possible instability in thermal behavior in the blends. For demonstration purposes, not all compositions were investigated; instead, only a 50/50 composition was chosen for aging at 25,30 , and $35^{\circ} \mathrm{C}$, respectively, for various times from 0 to $24 \mathrm{~h}$. Figure 3 shows the DSC traces of the physical-aged PTT/PBT blends (physically aged at $25^{\circ} \mathrm{C}$ for various times as indicated on the DSC traces). The physically aged blends exhibited a better-resolved $T_{\mathrm{g}}$, with an enthalpy relaxation

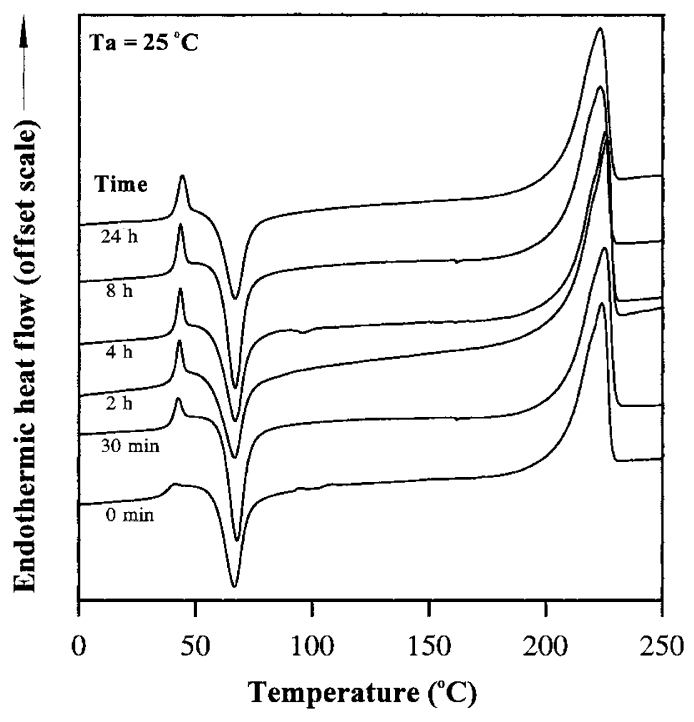

Figure 3. DSC traces of the physically aged PTT/PBT blends (aged at $25^{\circ} \mathrm{C}$ for various times as indicated on the DSC traces).

peak superimposed on the glass transition, and all the traces (for samples aged for different times) apparently showed only a single $T_{\mathrm{g}}$ for all compositions. Expectedly, the signal of the superimposed enthalpy relaxation peak increases in intensity with the time of aging; but all other thermal signals $\left(T_{\mathrm{g}}, T_{\mathrm{cc}}\right.$, and $\left.T_{\mathrm{m}}\right)$ remained about the same. In addition, a blend sample of 50/50 blend composition physically aged at two other temperatures ( 30 and $35^{\circ} \mathrm{C}$, respectively) for various times from 0 to $24 \mathrm{~h}$ yielded similar results, suggesting quite good consistency regardless of the aging temperatures imposed on the blend samples. For brevity, the results of physical aging of blend samples at two other temperatures are not shown here.

Thermal transitions in the blend other than $T_{\mathrm{g}}$ also provide clues of the extent or scales of molecular interactions, and extents of miscibility between two polyesters. Both the apparent $T_{\mathrm{m}}$ and peak position of cold crystallization exotherm can reflect if or not intimate molecular interactions exist in the polymer mixtures. Figure 4 shows the variation of the apparent $T_{\mathrm{m}}$ and cold crystallization peak $\left(T_{\mathrm{cc}}\right)$ of the PTT/PBT blends of all compositions investigated. Interestingly, the temperature locations of $T_{\mathrm{cc}}$ and $T_{\mathrm{m}}$ both change more rapidly for the intermediate compositions, but less so and stay quite stationary for the compositions near the two neat polymers. Note that there are two crystalline polymers in the blends, and thus, there should be two melting and crystallization peaks upon scanning. It may not be surprising that there is only one identifiable $T_{\mathrm{m}}$ for the PTT/PBT blends of all composition as the $T_{\mathrm{m}}$ 's for these two neat polymers (PTT and PBT) are quite close to each other, and therefore, peak merging might be possible. However, $T_{\mathrm{cc}}$ 's of these 


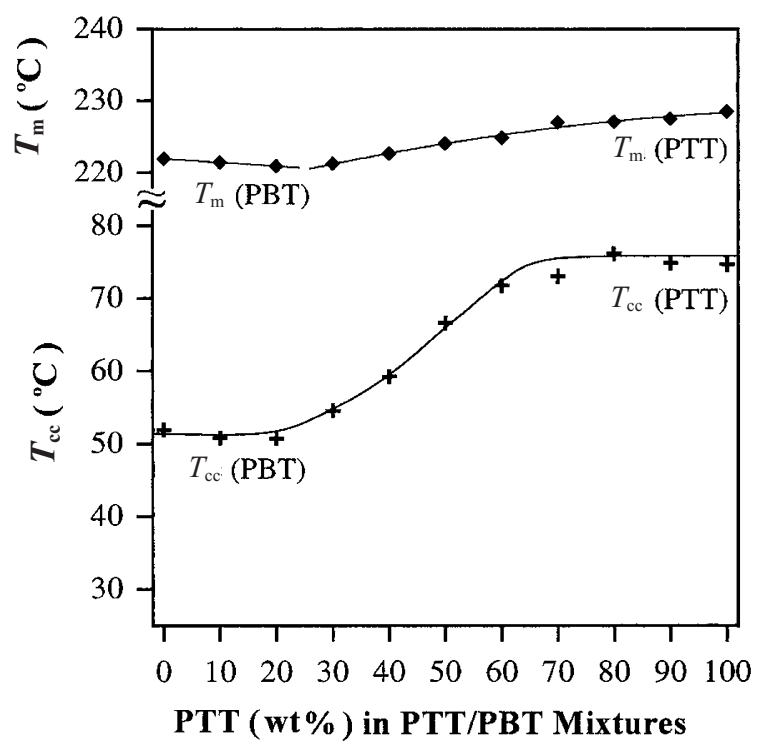

Figure 4. Variation of the apparent $T_{\mathrm{m}}$ and cold crystallization peak $\left(T_{\mathrm{cc}}\right)$ of the PTT/PBT blends with respect to compositions.

two neat polymers are widely different; nevertheless, there is only one composition-dependent $T_{\mathrm{cc}}$ peak for the blends of all compositions.

Thus, it is worth noting that there is only one single composition-dependent $T_{\mathrm{cc}}$ identified for each of the blend compositions. Recently, the crystallization peak $\left(T_{\mathrm{cc}}\right)$ in blends of two semicrystalline polymers has been proposed as an additional criterion for judging the miscibility and that a miscible mixture comprising both crystallizable polymers should exhibit a single $T_{\mathrm{cc}}$ peak as the quenched amorphous mixture is scanned. ${ }^{18}$ This is useful and convenient, especially for semicrystalline polymer blends whose constituents $T_{\mathrm{g}}$ 's are too closely spaced but the crystallization peak temperatures are sufficiently different. Thus, it can be taken as an indication that these two different chain segments in the miscibility state are intimately interacted and that they act in such concerted ways to exhibit only one thermal signal of $T_{\mathrm{cc}}$ during the event of crystallization from the rubber state above $T_{\mathrm{g}}$.

Crystallization Kinetics. Similarly, the crystallization behavior can be influenced by the miscibility of two different polymer chains. Crystallization halftimes may provide some additional evidence of miscibility of the two polymers in the blend. Figure 5 shows the crystallization half-times (at 195, 200, and $205^{\circ} \mathrm{C}$, respectively) as a function of PTT contents in the PTT/PBT blends. Within the temperature window examined, the $t_{1 / 2}$ for neat PTT, especially the half-time at $205^{\circ} \mathrm{C}$, is generally smaller than that for neat PBT. It is interesting that the half-times for the blends are roughly in between those for the neat polymers (PBT, PTT), with a slight deviation from the norm for the several compositions near the middle $(50 / 50)$. This be-

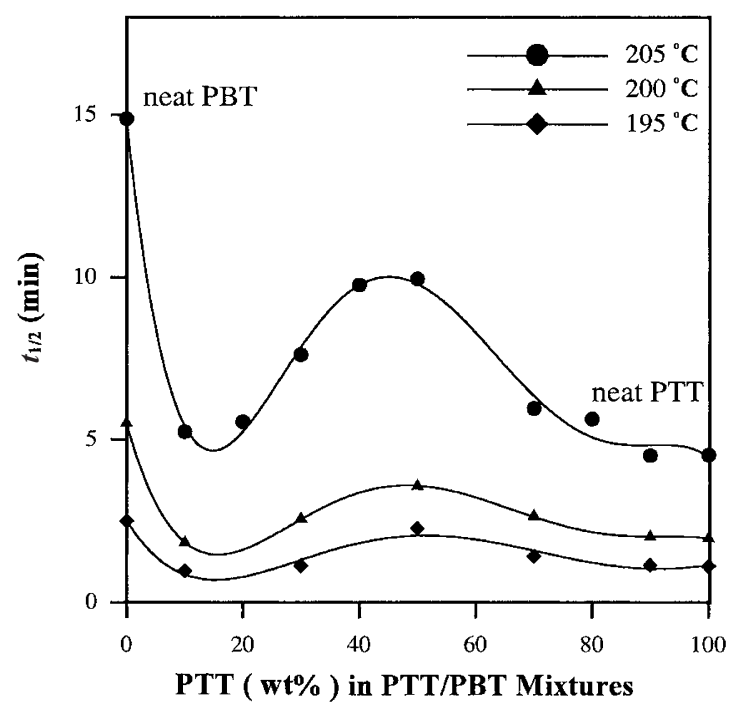

Figure 5. Crystallization half-times (at 195,200 , and $205^{\circ} \mathrm{C}$, respectively) as a function of PTT contents in the $\mathrm{PTT} / \mathrm{PBT}$ blend system.

havior suggests that that crystallization kinetics for the blend containing two miscible chain segments are an average of two neat polymers. It is known that a miscible blend can vary in the phase homogeneity with respect to the blend composition. The phase homogeneity and degree of chain inter-mixing near the middle composition (i.e., 50/50) are usually less in comparison to those near the two extreme compositions (e.g., 10/90 and 90/10). The deviation of the half-times for the PTT/PBT blends near 50/50 can be attributed to variation of the chain mixing state.

Figure 6 shows the X-Ray crystallographs for the neat polymers of PTT, PBT, and 50/50 blend. The neat PTT and PBT exhibited a few peaks that differed in the diffraction angles. The 50/50 blend exhibited both characteristic peaks of PTT and PBT, indicating that the blend contained both types of crystal cells. The POM result apparently showed that the crystalline region of the 50/50 blend contained only a single type of spherulites (rather than two different spherulites types attributable to the PTT and PBT crystals, respectively). Thus, more likely lamellae or lamellar bundles were composed of individually different unit cells of PTT and PBT but they grow into common spherulites. That is to say, within a single spherulite, two different unit cells may form individual lamellae, but individual lamellae co-exist in lamellar bundles.

Quenched samples of the PTT/PBT blends appeared optically clear as viewed using OM. In addition, SEM was further performed on the quenched blend samples. Quenching was intended to eliminate possible traces of crystals in the samples, which might cause haziness and complicated analysis of amorphous phase behavior of the blends. Figure 7 shows SEM graphs 


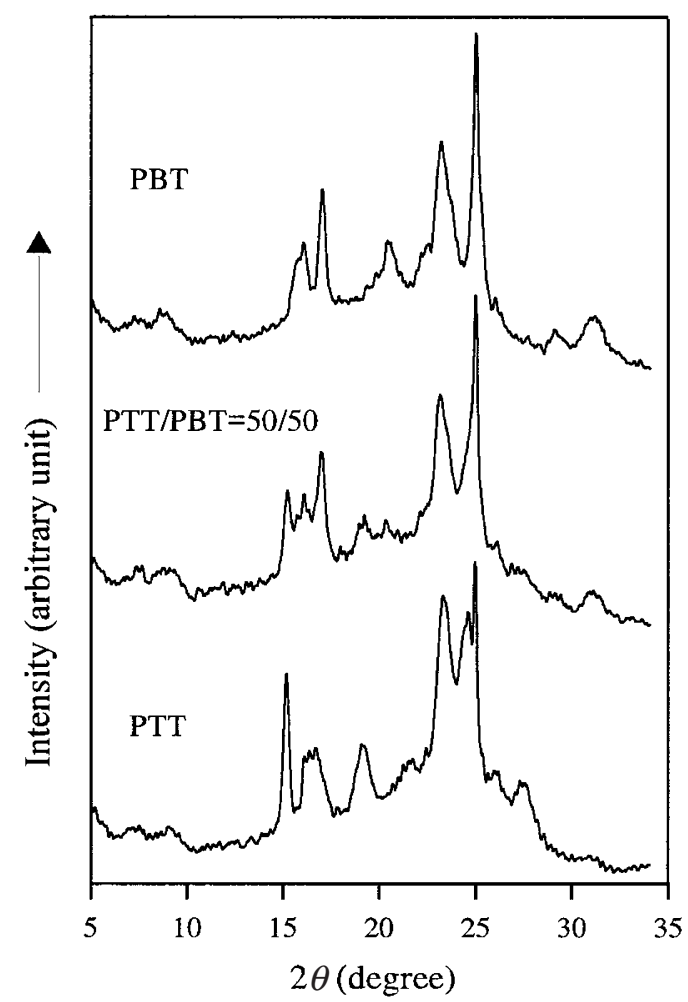

Figure 6. Comparison of X-Ray crystallographs for the neat PTT, PBT, and 50/50 blend.

for the PTT/PBT blend samples of compositions: (A) 90/10, (B) 70/30, (C) 50/50, (D) 30/70, and (E) 10/90 (weight ratios). SEM for the neat polymers (PTT, PBT) was also characterized, but for brevity, they are not all shown here. As shown in the graphs, all the samples exhibited a similarly homogeneous morphology free of any discernible separated domains. The graphs of blend morphology provide a supportive view of miscibility by revealing a homogeneous structure free of any separated phase domains at the feasible magnification of SEM.

PTT/PET Blend System. Thermal analysis was performed to reveal the thermal transitions of these blends. Figure 8 shows the DSC thermograms $\left(20^{\circ} \mathrm{C} \mathrm{min}^{-1}\right)$ revealing only a single $T_{\mathrm{g}}$ (arrow-marked) for each of the PTT/PET blend samples of 11 different compositions as indicated on the DSC traces. Again, the single $T_{\mathrm{g}}$ for each of the PTT/PET blend samples increases steadily with composition changes, i.e., it increases with the increase of the higher- $T_{\mathrm{g}}$ PET content in the blend. In addition, a cold-crystallization exotherm is apparent for all blend compositions. Interestingly, these two semicrystalline polymer chains in the blend seem to crystallize simultaneously upon heating, showing only a single exotherm peak. There are two trends for the crystallization exotherm of the blends, which varies systematically with respect to the compositions. The first trend is that the crystallization exotherm peak tem-
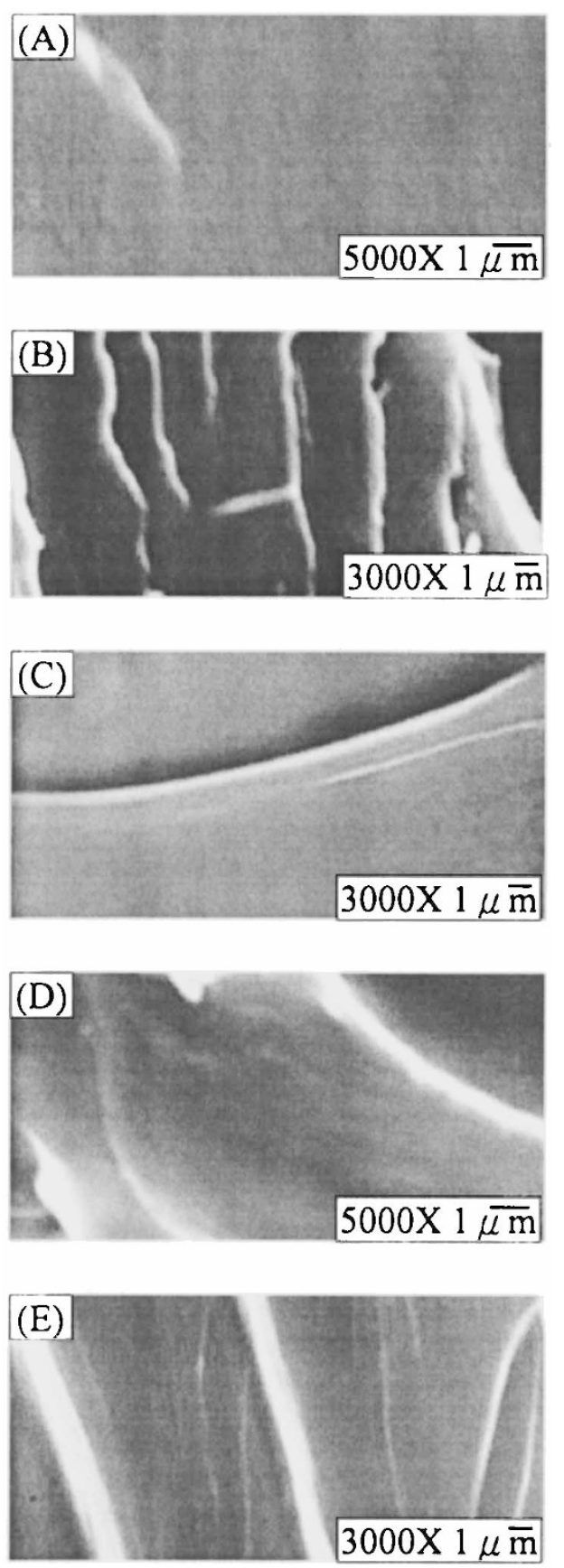

Figure 7. SEM graphs showing homogeneity and lack of any discernible domains in the fractured surfaces of the quenched PTT/PBT blends: (A) 90/10, (B) 70/30, (C) 50/50, (D) 30/70, and (E) $10 / 90$ (wt. ratios).

perature increases with the increase of PET contents, and the second trend is that the peak width gradually changes from the wider characteristic of neat PET for the PET-rich blends to narrow characteristic of neat PTT for the PTT-rich blends. These phenomena further indicate that that the polymers chain segments in the mixtures are likely intimately mixed in fine segmental scales and that these two intimately mixed chains may act as if the system exhibits only a single $T_{\mathrm{g}}$ in responding to external thermal changes. All these features suggest that the polymer chains in the PTT/PET 


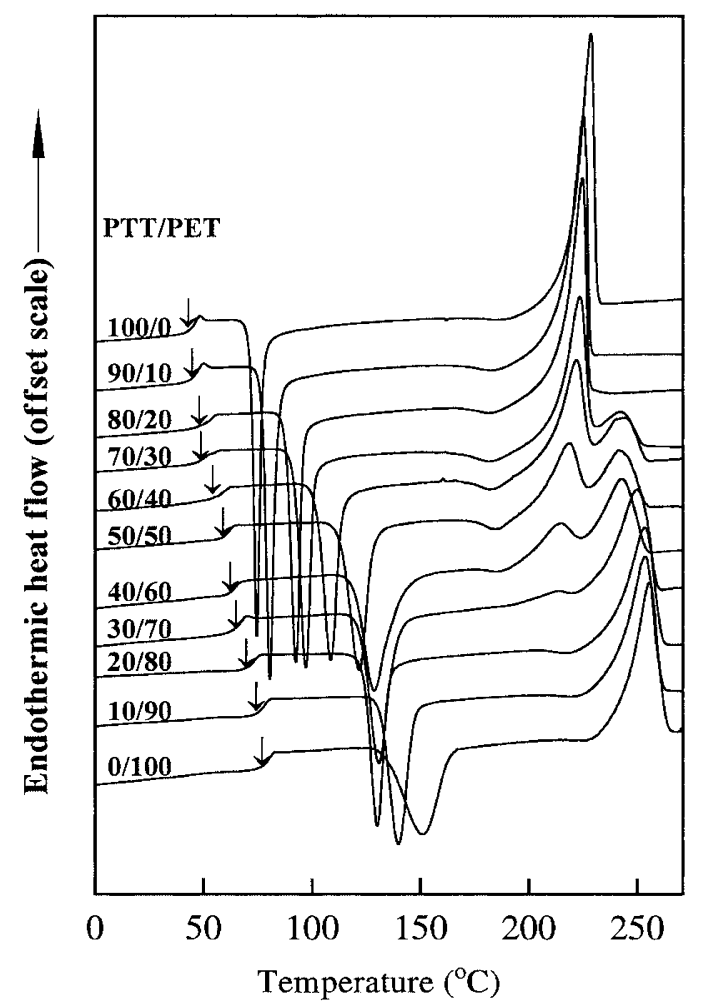

Figure 8. DSC thermograms for the PTT/PET blend samples of 11 different compositions.

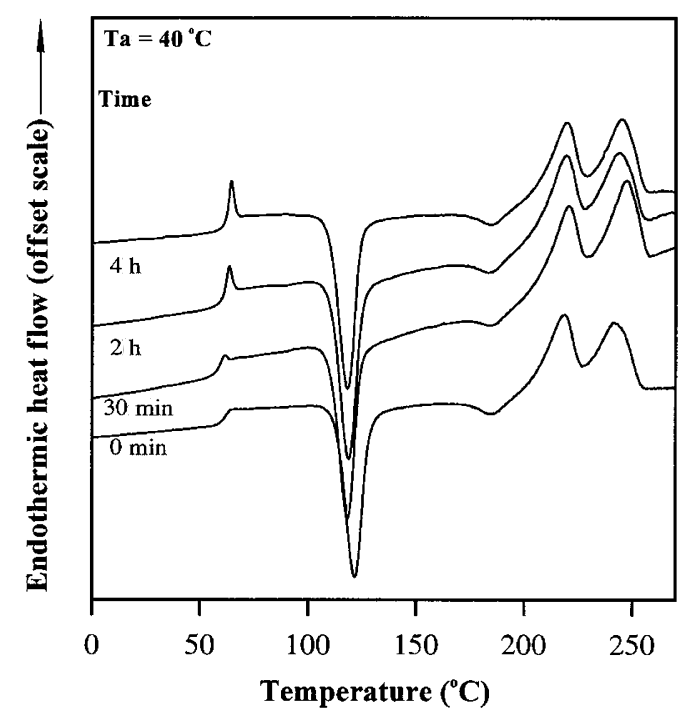

Figure 9. DSC traces of the physical-aged PTT/PET blends (aged at $40^{\circ} \mathrm{C}$ for various times as indicated).

blend system are intimately mixed in such fine molecular/segmental scales that the thermal responses of the mixtures act as averages of these two different polymers.

The main thermal characteristics were found to be quite similar for the PTT/PET and PTT/PBT blend systems. The $T_{\mathrm{g}}$ resolution was similarly enhanced by observing enthalpy relaxation peak(s) of the physicalaged blend samples. Figure 9 shows the DSC traces of the aged PTT/PET blends (50/50 composition, physi-

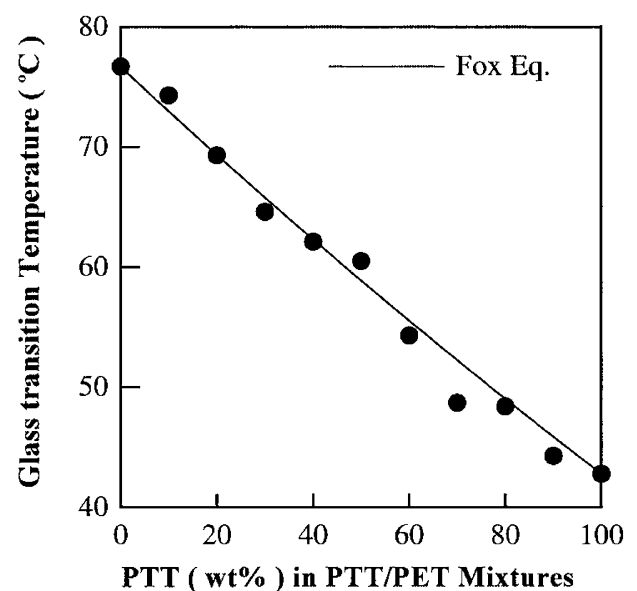

Figure 10. Plot of $T_{\mathrm{g}} v s$. composition for PTT/PET blend system.

cally aged at $40^{\circ} \mathrm{C}$ for various times as indicated on the DSC traces). With the enhanced resolution and better signal, the DSC result for the PTT/PET blend sample apparently confirmed a single $T_{\mathrm{g}}$.

Figure 10 shows a plot of $T_{\mathrm{g}} v s$. composition relationship for the PTT/PET blend system. Interestingly, the earlier discussed PTT/PBT blend showed a $T_{\mathrm{g}}$-composition relationship in agreement with the Gordon-Taylor (G-T) equation: ${ }^{17} T_{\mathrm{g}}=\left(\omega_{1} T_{\mathrm{g} 1}+\mathrm{k}\right.$ $\left.\omega_{2} T_{\mathrm{g} 2}\right) /\left(\omega_{1}+\mathrm{k} \omega_{2}\right)$, where $\omega_{i}$ is the mass fraction of polymer component $\mathrm{i}$, and $\mathrm{k}$ is a parameter. But the experimental $T_{\mathrm{g}}-v s$.-composition for the PTT/PET blend system as shown is in agreement with the Fox equation (1/ $\left.T_{\mathrm{g}}=\omega_{1 /} T_{\mathrm{g} 1}+\omega_{2 /} T_{\mathrm{g} 2}\right)$. This fact suggests that it is a miscible blend system whose phase homogeneity is reasonably close to the segmental homogeneity that is commonly seen in covalent-bonded copolymers of two monomer units

Figure 11 shows the variation of the apparent $T_{\mathrm{m}}$ and cold crystallization peak $\left(T_{\mathrm{cc}}\right)$ of the PTT/PET blends of all compositions investigated. Note that there are two crystalline polymers in the blends, and thus, there should be two melting and crystallization peaks upon DSC scanning. However, there is only one $T_{\mathrm{cc}}$ identified for each of the blend compositions. The peak temperature of the single $T_{\mathrm{cc}}$ changed almost proportionally with the blend composition. The fact suggests two possibilities that either two peaks are partially overlapped into an apparent peak, or these two molecular chain segments in the blend act in such concerted ways that there is only one thermal signal for these two polymer segments. The single $T_{\mathrm{cc}}$ peak for the blends indicated that upon crystallization, these two different chain segments in the blend act in concerted ways to exhibit only one thermal signal (i.e., a single $\left.T_{\text {cc }}\right)$. The polymer chains, however, were packed into two different crystal cell types individually in the com- 


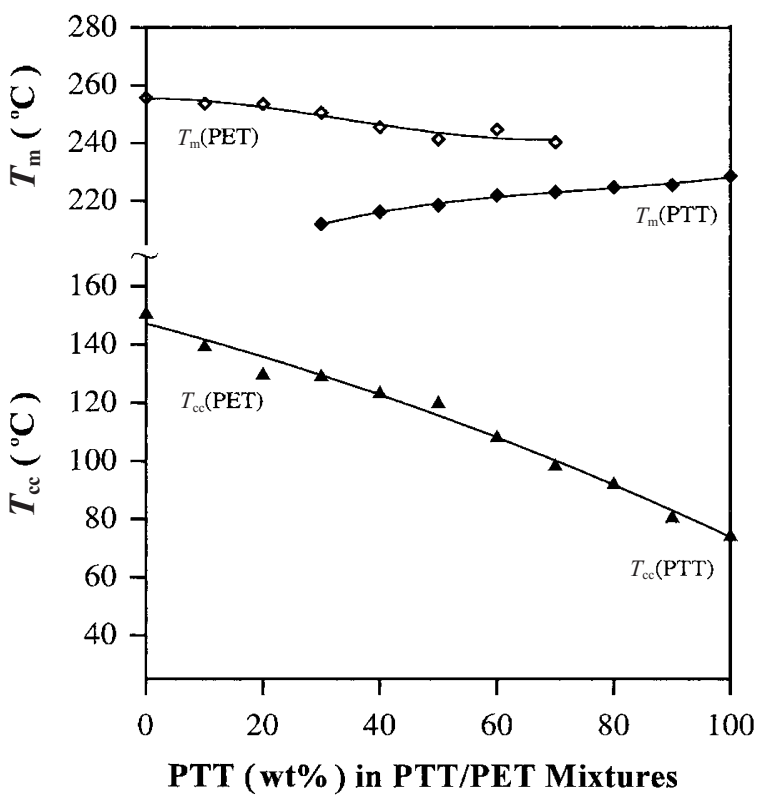

Figure 11. Variation of the apparent $T_{\mathrm{m}}$ and cold crystallization peak $\left(T_{\mathrm{cc}}\right)$ of the PTT/PET blends with respect to compositions.

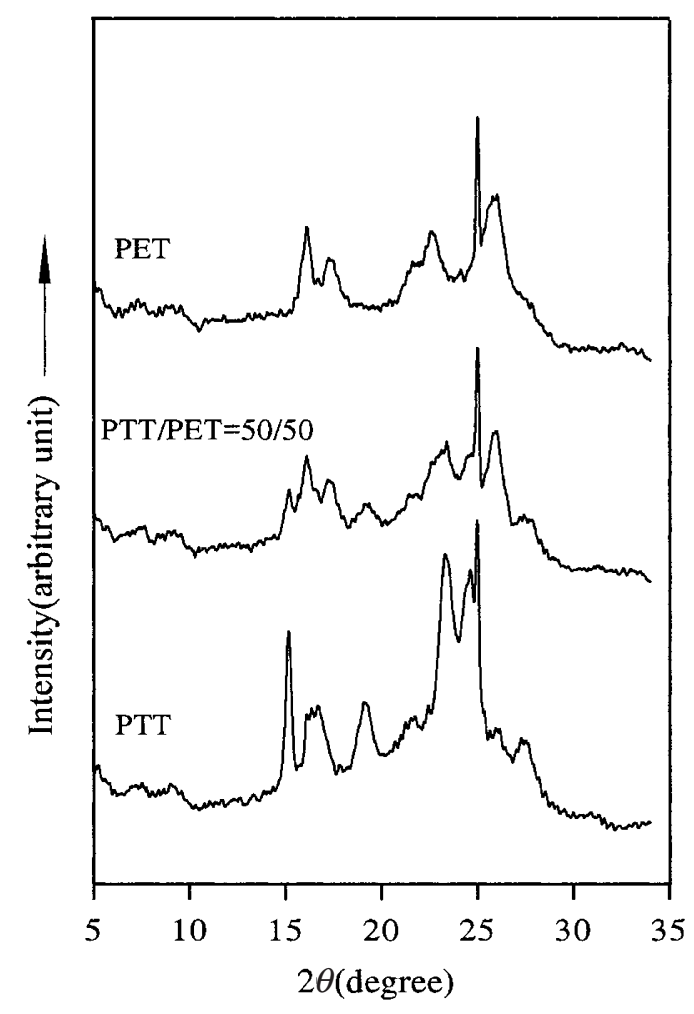

Figure 12. X-Ray crystallographs (top to bottom) for neat PET, 50/50 PTT/PET blend, and neat PTT.

mon spherulites. This fact is clearly seen in these two melting peaks for most of the intermediate blend compositions. Note that each of the melting peak temperatures (for the PTT component in the PTT-rich blend and the PET component in the PET-rich blends) is depressed with the greater content of the other polymer in the blend, suggesting influence of the miscibility in the
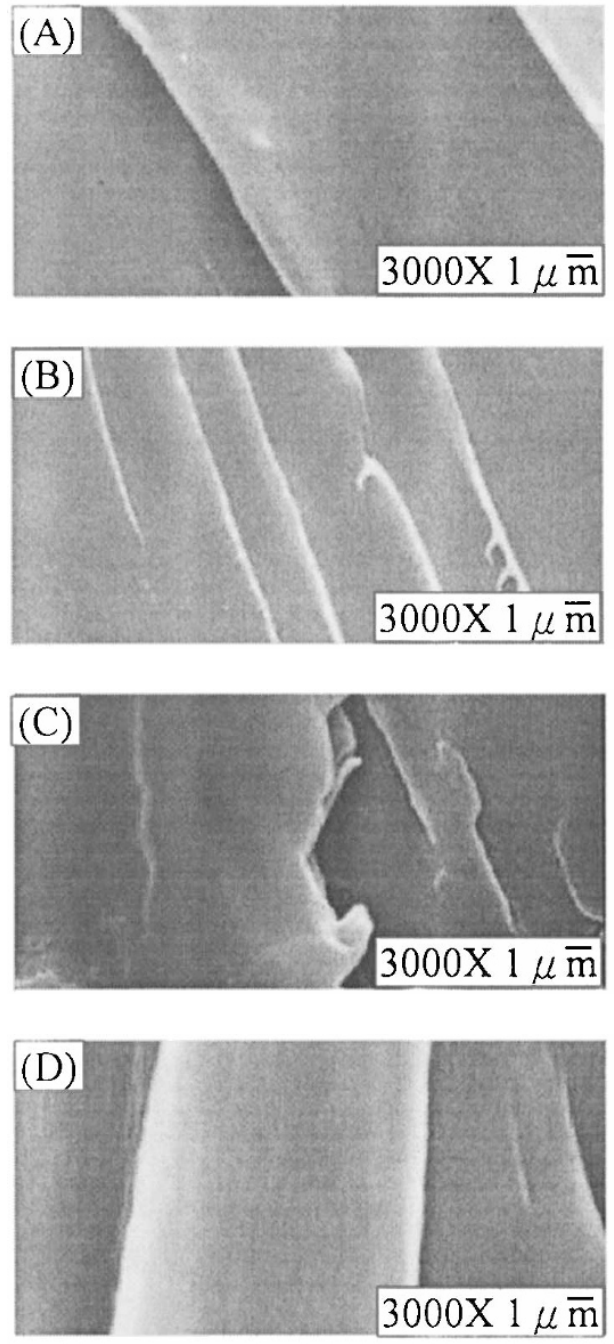

Figure 13. SEM graphs showing homogeneity and lack of any discernible domains in the fractured surfaces of the quenched PTT/PET blends: (A) 90/10, (B) 70/30, (C) 30/70, and (D) 10/90 (wt. ratios).

crystalline-crystalline polymer blends.

Figure 12 shows the X-Ray crystallographs for the neat polymers of PTT, PET, and 50/50 PTT/PET blend. The neat PTT and PET exhibited a few diffraction peaks that differed in the diffraction angles. The 50/50 blend exhibited both characteristic peaks of PTT and PET, indicating that the blend contained both types of crystal cells. The result confirmed that the crystal types in the miscible blend remained individually different, and that miscibility is restricted to the amorphous region of the crystalline/crystalline blend. The microscopy result also revealed a homogeneous morphology in the blend system of two polyesters. Quenched samples of the PTT/PET blends appeared optically clear as viewed using OM. Similarly, SEM was further performed on the quenched blend samples. Figure 13 shows SEM graphs for the PTT/PET blend samples: (A) $90 / 10$, (B) 70/30, (C) 50/50, and (D) 30/70, (E) 10/90 (weight ratios in blend compositions). 
The graphs for the blend morphology have provided a supportive view of miscibility by revealing a homogeneous structure free of any separated phase domains at the feasible magnification of SEM.

\section{CONCLUSION}

Thermal, microscopy, and X-Ray characterizations were performed on two binary blend systems comprising two of three homologous and crystalline aryl-polyesters, namely, poly(ethylene terephthalate) (PET), poly(trimethylene terephthalate) (PTT), and poly(butylene terephthalate) (PBT). Two binary pairs, PTT/PBT and PTT/PET, were investigated in this study and were found to be both miscible using criteria of thermal analysis, glass transition behavior, microscopy, and crystallization kinetics. To ensure that physically blended mixtures of the aryl polyesters solution blending was used in comparison with melt blending. The solution mixing and preparation of the samples assured the absence of possible trans-esterification reactions that might occur in polyesters at high temperatures for extended times. In addition, considering that ambiguity of the glass transition criterion might arise owing to proximity of $T_{\mathrm{g}}$ 's of the constituent polymers, a technique of physical aging (annealing at below but near $T_{\mathrm{g}}$ ) was used to enhance the $T_{\mathrm{g}}$ resolution in assessing the thermal criteria of polymer miscibility. The aged blend samples exhibited distinctly single $T_{\mathrm{g}}$ (enthalpy relaxation peak) regardless of aging time and temperatures imposed, confirming phase stability and miscibility of the blends. In addition, only a single composition-dependent $T_{\mathrm{cc}}$ was identified for each of the blends comprising two crystallizable polymers. This additional evidence was also taken as a valid supportive evidence for miscibility in the blends of the aryl polyester polymers.

The miscibility, glass transition, and phase behavior of these two binary blend systems (PTT/PBT and PTT/PET, respectively) was found to be quite comparatively similar. The claimed miscibility applied to the quenched amorphous states of the blends (in absence of any discernible trans-esterifications), and not the crystallized states involving the crystal cells in the crystalline regions. As a matter of fact, this study demonstrated that during crystallization, the polymer chains of different segments might co-crystallize in the same bundles and same spherulites, but the crystal cell types remained individually different. For the crystalline/crystalline blend systems of PTT/PBT or PTT/PET, the miscibility is restricted to the amorphous region and the crystalline region consists of distinctly different unit cells in the same spherulites.

Acknowledgment. The grant (No. NSC-89-2218E006-035) is generously provided by Taiwan's $\mathrm{Na}$ tional Science Councilin consecutive years. The authors also acknowledge the kind supply of research polymer materials from ITRI (Taiwan) and GE Corp. (USA), respectively.

\section{REFERENCES}

1. S. Saeki, J. M. G. Cowie, and I. J. McEwen, Polymer, 24, 60 (1983).

2. J. M. Widmaier and G. Mignard, Eur. Polym. J., 23, 989 (1987).

3. J.-L. Lin and R.-J. Roe, Macromolecules, 20, 218 (1987).

4. H. A. Schneider and P. Dilger, Polym. Bull. 21, 265 (1989).

5. A. Rameau, Y. Gallot, P. Marie, and B. Farnoux, Polymer, 30, 386 (1989).

6. T. A. Callaghan and D. R. Paul, Macromolecules, 26, 2439 (1993).

7. L. L. Chang and E. M. Woo, Macromol. Chem. Phys., 202, $636(2001)$

8. L. L. Chang and E. M. Woo, Macromolecules, 33, 6892 (2000).

9. C. Nakafuku and M. Sakoda, Polym. J., 25, 909 (1993).

10. C. Nakafuku, Polym. J., 28, 568 (1996).

11. I. Avramov and N. Avramova, J. Macromol. Sci., Phys. B30, 335 (1991).

12. N. Avramova, S. Fakirov, and I. Avramov, Die Angew. Makromol. Chem., 199, 129 (1992).

13. S. Fakirov, M. Evstatiev, and S. Petrovich, Macromolecules, 26, 5219 (1993).

14. N. Avramova, Polymer, 36, 801 (1995).

15. Y. Yu and K. J. Choi, Polym. Eng. Sci., 37, 91 (1997).

16. J. Font, J. Muntasell, and E. Cesari, Mater. Res. Bull., 34, 157 (1999).

17. M. Gordon and J. S. Taylor, J. Appl. Chem., USSR, 2, 493 (1952).

18. Y. H. Kuo, MS Thesis in Department of Chemical Engineering, National Cheng Kung University, Tainan, Taiwan, 2002. 